\title{
Relativistic meson-proton and proton-proton cross- sections and quantum molecular dynamics model
}

\author{
Ahmed Mohamed Abdalla \\ Department of Mathematical and Physical Engineering, Faculty of Engineering in Shoubra, Benha University, Cairo, Egypt \\ Email address: \\ a_abdalla65@hotmail.com
}

To cite this article:

Ahmed Mohamed Abdalla. Relativistic Meson-Proton and Proton-Proton Cross- Sections and Quantum Molecular Dynamics Model. International Journal of High Energy Physics. Vol. 1, No. 1, 2014, pp. 6-12. doi: 10.11648/j.ijhep.20140101.12

\begin{abstract}
Above $10^{4} \mathrm{GeV} / \mathrm{c}$ projectile momenta, the total cross-sections for $p p$ and $\bar{p} p$ collisions as a function of laboratory beam momentum and center of mass energy, are independent. In the range, 0.1 up to $10^{4} \mathrm{GeV} / \mathrm{c}$ the total cross-section depends on the nature of the interacting particles and on the geometrical interpretations in terms of the impact parameter. This could explain in terms of the Quantum Molecular Dynamic QMD model and its ultra relativistic approach UrQMD, in terms of the isospin of the colliding particles, their flavor, and center of mass energies. The model can give also a qualitative success for such dependencies for $\pi^{+} p$ and $k^{+} p$ interactions.
\end{abstract}

Keywords: Total Cross-Section, Meson, Proton, Collisions

\section{Introduction}

High-energy nucleon-nucleon and nucleus-nucleus collisions are the excellent tool to study nuclear matter under extreme conditions of temperature and density. There is great importance by production of charged particles in elementary proton-proton collisions and in heavy ion collisions. This allows for a first exploration of parton densities in the early stage and provides stringent limits for nearly all available theoretical models. It directly reflects how much of the initial beam energy can be converted to new particles and it is therefore directly linked to the stopping mechanism of the initial protons and nucleons. Thus, the particle multiplicity contains information about the entropy of the system and the gluon density in the first stage of the collision. The investigation of particle production in proton-proton collisions at Large Hadron Collider energies at CERN, LHC are expect to yield new insights into the underlying partonic processes. Data from the experimental collaborations [1-5] are now available starting at the injection energy of $\sqrt{s}=0.9 \mathrm{TeV}$, via $2.36 \mathrm{TeV}$, to the current maximum energy of seven $\mathrm{TeV}$. There are valuable and recent models give an inclusive description of the mechanism of reaction for hadron-hadron (h-h), and extended to nucleus-nucleus (A-A) interactions. In this work we will concern by the microscopic transport model called Quantum Molecular Dynamics (QMD) model [6-11].

QMD depends on the theory of N-body and simulates heavy ion reactions at intermediate energies basis on an event by event. Taking into account all fluctuations and correlations have two advantages first many body processes, in particular the formation of complex fragments are explicitly treated and second the model event by event analysis of heavy ion reactions. The important point for any theoretical model is making a comparison between the data obtained due to formal treatment of the model assumptions and the experimental results. The major aspects of the formulation of QMD will now discuss briefly in section 2 . For more detailed description in ref. [6]. The relativistic treatment for total cross-section will discuss in section 3 . The comparison with the recent results for baryon-baryon results will be in section 4 .

\section{Quantum Molecular Dynamic (QMD)}

The Quantum Molecular Dynamics QMD model describes the projectile and target nuclei, which are modeled according to the Fermi-gas ansatz. The nucleons are represented by Gaussian shaped density distributions (we set $\hbar, c=1)$ :

$$
\emptyset_{j}\left(\boldsymbol{x}_{\boldsymbol{j}}, t\right)=\left(\frac{2 \alpha}{\pi}\right)^{3 / 4} \exp \left\{-\alpha\left(x_{j}-r_{j}(t)\right)^{2}+\frac{i}{\hbar} p_{j}(t) x_{j}\right\}
$$

which is characterized by 6 time-dependent parameters, $q_{i}$ and $p_{i}$, respectively. The total $\mathrm{n}$-body wave function is assumed the direct product of single coherent states of 
nucleon Gaussian distribution given in Eq. (2.1)

$$
\Phi=\prod_{i} \emptyset_{i}\left(x, q_{i}, p_{i}, t\right)
$$

The initial values of the parameters are chosen in a way that the ensemble of $A_{T}+A_{P}$ nucleons gives a proper density distribution as well as a proper momentum distribution of projectile and target nuclei. The equations of motion of the many body system is calculated by means of a generalized variational principle we start out from the action

$$
S=\int_{t_{1}}^{t_{2}} \mathcal{L}\left[\Phi, \Phi^{*}\right] d t
$$

with the Lagrange functional $\mathcal{L}$

$$
\mathcal{L}=\left\langle\Phi\left|i \hbar \frac{d}{d t}-H\right| \Phi\right\rangle
$$

where the total time derivative includes the derivation with respect to the parameters. The Hamiltonian $H$, contains a kinetic term and mutual Coulomb interactions $V_{i j}$. The time evolution of the parameters is obtained by the requirement that the action is stationary under the allowed variation of the wave function. This yields an Euler-Lagrange equation for each parameter. For the coherent states and a Hamiltonian of the form

$$
H=\sum_{i} T_{i}+\frac{1}{2} \sum_{i j} V_{i j}
$$

$\left(\mathrm{T}_{\mathrm{i}}=\right.$ kinetic energy, $V_{i j}=$ potential energy $)$ the Lagrangian and the variation can easily calculate and we obtain:

$$
\begin{gathered}
\mathcal{L}=\sum_{i}\left[-\dot{q}_{i} p_{i}-T_{i}-\frac{1}{2} \sum_{j \neq i}\left\langle V_{i k}\right\rangle-\frac{3}{2 L m}\right] \\
\dot{q}_{i}=\frac{p_{i}}{m}+\nabla p_{i} \sum_{j}\left\langle V_{i k}\right\rangle=\nabla p_{i}\langle H\rangle \\
\dot{p}_{i}=-\nabla q_{i} \sum_{j \neq i}\left\langle V_{i k}\right\rangle=-\nabla q_{i}\langle H\rangle
\end{gathered}
$$

with $\left\langle V_{i j}\right\rangle=\int d^{3} x_{1} d^{3} x_{2} \Phi_{i}^{*} \Phi_{j}^{*} V\left(x_{1}, x_{2}\right) \Phi_{i} \Phi_{j}$

These are the time evolution equations, which solved numerically. Thus the variational principle reduces the time evolution of the n-body Schrödinger equation to the time evolution equations of $6\left(A_{P}+A_{T}\right)$ parameters to which a physical meaning can be attributed. The equations of motion for the parameters $p_{i}$ and $q_{i}$ read and show the same structure as the classical Hamilton equations. The numerical solution can be treated in a similar manner as it is done in classical molecular dynamics [12-6]. Using tria-wave functions other than Gaussians in Eq.(1.1) yields more complex equations of motion and hence the analogy to classical molecular dynamics is lost. If $\langle H\rangle$ has no explicit time-dependence, QMD conserves energy and momentum by construction.

$$
\dot{p}_{i}=-\frac{\partial\langle H\rangle}{\partial q_{i}} \quad \text { and } \quad \dot{q}_{i}=-\frac{\partial\langle H\rangle}{\partial p_{i}}
$$

The Relativistic Quantum Molecular Dynamics (RQMD)approach has been developed to extend the QMD model up to relativistic energies (AGS and CERN/SPS domain) [17].
Its main improvements compared to the standard QMD model [6-9,18-20] are 1.covariant dynamics 2. an improved and extended collision term containing heavy baryon resonances, strange particles and string excitation for high energy hadron-hadron interactions.

Each initialized nucleus must meet the following constraints:

- $\sum_{i} q_{i}=0$, i.e. it is centered in configuration space around 0 ,

- $\sum_{i} v_{i}=0$, i.e. the nucleus is at rest

- its binding energy should correspond to the value given by the Bethe-Weizsäcker formula,

- the radius should yield the following mass dependence

$$
R(A) \sim r_{0} \cdot A^{1 / 3}
$$

and have a reasonable surface-thickness,

- in its center, the nucleus should have nuclear matter ground-state density.

In configuration space, the centroids of the Gaussians are randomly- distributed within a sphere. The finite width of Gaussians results in a surface region beyond the radius of that sphere. Therefore, its radius is reducing by half a layer of nucleons from the original nuclear radius of equation (2.9):

$$
R(A)=r_{0}\left(\frac{1}{2}\left[A+\left(A^{1 / 3}-1\right)^{3}\right]\right)^{1 / 3}
$$

The parameter $r_{0}$ is a function of the nuclear matter ground state density $\rho_{\mathrm{o}}$ used in the UrQMD model:

$$
r_{0}=\left(\frac{3}{4 \pi \rho_{0}}\right)^{1 / 3}
$$

The relatively small number of nucleons can be distributed over the volume of the nucleus may result in large fluctuations in the mean density of the nucleus. Therefore, the phase-space density at the location of each nucleon evaluated after its placement. If the phase-space density is too high, (i.e. other nucleons occupy the respective area of the nucleus.), then the location of that nucleon is rejected and a new location is randomly chosen. The initial momenta of the nucleons are randomly chosen between 0 and the local Thomas-Fermi momentum:

$$
p_{F}^{\max }=\hbar c\left(3 \pi^{2} \rho\right)^{1 / 3}
$$

with $\rho$, being the corresponding local proton or neutron-density.

The real part of the nucleon-nucleon interaction as it is implemented into the UrQMD model [21] is based on a non-relativistic density-dependent Skyrme-type equation of state with additional Yukawa and Coulomb potentials. Momentum dependent potentials are not used a Pauli-potential, however, may be included optionally. The nucleon- or baryon-density can be obtained from the Gaussian (2.1):

$$
\varrho\left(x_{j}, t\right)=\left(\frac{2 \alpha}{\pi}\right)^{3 / 2} \exp \left\{-2 \alpha\left(x_{j}-r_{j}(t)\right)^{2}\right\}
$$


where $x_{j}$ denotes the quantum mechanical position variable, while $r_{j}(t)$ is the classical parameter of the Gaussian. The Skyrme-Potential (momentum-dependence and spin-exchange has been neglected) has the form:

$$
V^{S K}=\frac{1}{2 !} t_{1} \sum_{j, k}^{\prime} \delta\left(x_{j}-x_{k}\right)+\frac{1}{3 !} t_{2} \sum_{j, k, l}^{\prime} \delta\left(x_{j}-x_{k}\right) \delta\left(x_{j}-x_{l}\right)
$$

In order to avoid self-interactions, all terms where at least two indices are identical are discarding in the primed sum. This potential consists of a sum of two and a three body interaction terms. The two-body term, which has linear density dependence, models the long-range attractive component of the nucleon-nucleon interaction, whereas the three-body term with its quadratic density dependence is responsible for the short-range repulsive part of the interaction. Using the Gaussian (2.1) as the wave function of the nucleon, we obtain for the two-body Skyrme potential of particle $\mathrm{j}$ :

$$
\begin{gathered}
V_{j}^{S K 2}=\sum_{\left.-x_{k}\right)}^{\sum_{j}^{\prime} \varphi_{j}\left(x_{j}\right) \varphi_{k}\left(x_{k}\right)} x_{j} d x_{k} \varphi_{j}^{*}\left(x_{j}\right) \varphi_{k}^{*}\left(x_{k}\right) t_{1} \delta\left(x_{j}\right. \\
=t_{1} \sum_{K}^{N^{\prime}}\left(\frac{\alpha}{\pi}\right)^{\frac{3}{2}} \exp \left\{-\alpha\left(r_{j}-r_{k}\right)^{2}\right\} \\
=t_{1} \varrho_{j}^{i n t}\left(r_{j}\right)
\end{gathered}
$$

In the last line the interaction density was introduced. This density has the same form as the nucleon density (2.13) obtained from the Wigner-transform of the Gaussian (2.1), but omits the nucleon at the location $\mathrm{j}$ and its Gaussian has twice the width of that used in equation (2.13). The three-body potential for particle $\mathrm{j}$ can be derived in an analogous fashion:

$$
\begin{gathered}
V_{j}^{S K 3}=\frac{1}{2 !} \sum_{k l}^{N^{\prime}} \int d x_{j} d x_{k} d x_{l} \varphi_{j}^{*}\left(x_{j}\right) \varphi_{k}^{*}\left(x_{k}\right) \varphi_{l}^{*}\left(x_{l}\right) \\
\times t_{2} \delta\left(x_{j}-x_{k}\right) \delta\left(x_{j}-x_{l}\right) \varphi_{j}\left(x_{j}\right) \varphi_{k}\left(x_{k}\right) \varphi_{l}\left(x_{l}\right) \\
=t_{2} \frac{1}{2 !} \sum_{K l}^{N^{\prime}}\left(\frac{4 \alpha^{2}}{3 \pi^{2}}\right)^{\frac{3}{2}} \exp \left\{-\frac{2}{3} \alpha\left(\left(r_{j}-r_{k}\right)^{2}+\left(r_{k}-r_{l}\right)^{2}+\right.\right. \\
\left.\left.\left(r_{l}-r_{j}\right)^{2}\right)\right\}
\end{gathered}
$$

In the case of infinite nuclear matter the individual relative distances should be close to their average value. Therefore the relative distance between particle $k$ and $l$ may be substituted by the average of the other two relative distances:

$$
\begin{gathered}
V_{j}^{S K 3} \approx \frac{1}{2 !} t_{2} \sum_{K l}^{N \prime}\left(\frac{4 \alpha^{2}}{3 \pi^{2}}\right)^{\frac{3}{2}} \exp \left\{-\alpha\left(\left(r_{j}-r_{k}\right)^{2}+\right.\right. \\
\left.\left.\left(r_{j}-r_{l}\right)^{2}\right)\right\}
\end{gathered}
$$

Using the definition of the interaction-density given in equation (2.15), the quadratic density dependence of the three-particle term (2.17) may generalize to arbitrary exponents for the density. This is of great importance for the implementation of a so-called soft equation of state. Then, however, the interpretation of $V_{j}^{S K 3}$ as three-particle interaction is no longer valid:

$$
V_{j}^{S K 3} \approx t_{2} 3^{-\frac{3}{2}}\left(\varrho_{j}^{i n t}\right)^{2} \rightarrow t_{\gamma}(\gamma+1)^{-3 / 2}\left(\varrho_{j}^{i n t}\right)^{\gamma}
$$

In the UrQMD model expression (2.18) is always used, even for the case $\gamma=2$.

The Yukawa-, Coulomb-, and (optional) Pauli-potentials may be written in the form of two-particle interactions:

$$
\begin{gathered}
V_{\text {Yuk }}^{i j}=V_{0}^{\text {Yuk }} \frac{\exp \left\{-\left|r_{i}-r_{j}\right| / \gamma_{Y}\right\}}{\left|r_{i}-r_{j}\right|} \\
V_{\text {Coul }}^{i j}=\frac{z_{i} z_{j} e^{2}}{\left|r_{i}-r_{j}\right|} \\
V_{\text {Pau }}^{i j}=V_{\text {Pau }}^{0}\left(\frac{\hbar}{q_{0} p_{0}}\right)^{3} \exp \left\{-\frac{\left|r_{i}-r_{j}\right|^{2}}{2 q_{0}^{2}}-\frac{\left|p_{i}-p_{j}\right|^{2}}{2 q_{0}^{2}}\right\} \delta_{\tau_{i} \tau_{j}} \delta_{\sigma_{i} \sigma_{j}}
\end{gathered}
$$

$\sigma_{j}$ and $\tau_{j}$ denote the spin and isospin of particle $j$ and $Z_{i}$ represents its charge. In infinite nuclear matter, the contribution of the Yukawa-potential to the total energy has a linear density-dependence, just like the two-body Skyrme-contribution. Therefore, all parameter sets, which satisfy the following relation for the parameter $t_{1}$, yield the same equation of state in infinite nuclear matter:

$$
\frac{1}{2} t_{1}+2 \pi V_{0}^{Y u k} \gamma_{Y}^{2}=\text { const }
$$

In finite nuclei, the usage of a Yukawa-potential has the advantage that the parameters can be tuned to the proper surface properties of the nuclei without changing the equation of state. The classical UrQMD Hamiltonian, which governs the motion of the parameters $r_{i}$ and $p_{j}$ of the wave functions, is thus given by:

$$
\begin{gathered}
H_{U r Q M D}=\sum_{J=1}^{N} E_{j}^{\text {Kin }}+\frac{1}{2} \sum_{j=1}^{N} \sum_{k=1}^{N}\left(E_{j k}^{S k 2}+E_{j k}^{\text {Yukawa }}+\right. \\
\left.E_{j k}^{\text {Couomb }}+E_{j k}^{\text {Pauli }}\right)+\frac{1}{6} \sum_{j=1}^{N} \sum_{k=1}^{N} \sum_{l=1}^{N} E_{j k l}^{S k 3}
\end{gathered}
$$

The individual contributions are defined as:

$$
\begin{gathered}
E_{j}^{K i n}=\sqrt{p_{j}^{2}+m_{j}^{2}}, \\
E_{j k}^{S k 2}=t_{1}\left(\frac{\alpha}{\pi}\right)^{3 / 2} \exp \left\{-\alpha r_{j k}^{2}\right\}, \\
E_{j k l}^{S k 3}=t_{\gamma}\left(\frac{4 \alpha^{2}}{3 \pi^{2}}\right)^{3 / 2} \exp \left\{-\alpha\left(r_{j k}^{2}+r_{j l}^{2}\right)\right\}, \\
E_{j k}^{\text {Yukawa }}=V_{0}^{Y u k} \frac{1}{2 r_{j k}} \exp \left\{\frac{1}{4 \alpha \gamma_{Y}^{2}}\right\}\left[\exp \left\{-\frac{r_{j k}}{\gamma_{Y}}\right\} \times\right. \\
\left(1-\operatorname{erf}\left(\frac{1}{2 \gamma_{Y} \sqrt{\alpha}}-\sqrt{\alpha} r_{j k}\right)\right)-\exp \left\{\frac{r_{j k}}{\gamma_{Y}}\right\} \times(1- \\
\left.\left.\operatorname{erf}\left(\frac{1}{2 \gamma_{Y} \sqrt{\alpha}}+\sqrt{\alpha} r_{j k}\right)\right)\right] \\
E_{j k}^{\text {Coulomb }}=\frac{z_{i} Z_{j} e^{2}}{r_{j k}} \operatorname{erf}\left(\sqrt{\alpha} r_{j k}\right),
\end{gathered}
$$




$$
\begin{gathered}
E_{j k}^{\text {Pauli }}=V_{0}^{\text {Pau }}\left(\frac{\hbar}{p_{0} q_{0}}\right)^{3}\left(1+\frac{1}{2 \alpha q_{0}^{2}}\right)^{-3 / 2} \exp \left\{-\frac{\alpha r_{j k}^{2}}{2 \alpha q_{0}^{2}+1}-\right. \\
\left.\frac{p_{j k}^{2}}{2 p_{0}^{2}}\right\} \delta_{\tau_{j} \tau_{k}} \delta_{\sigma_{j} \sigma_{k}}
\end{gathered}
$$

with $\quad r_{j k}=\left|r_{j}-r_{k}\right|$ and $\quad p_{j k}=\left|p_{j}-p_{k}\right|$

So far, only a hard equation of state has been implemented into the UrQMD model. The respective parameters are listed in table 1.

Table 1. Parameters of the hard equation of state implemented in UrQMD model, with and without Pauli-potential.

\begin{tabular}{ccc}
\hline parameter & $\begin{array}{c}\text { without } \\
\text { Pauli-potential }\end{array}$ & $\begin{array}{c}\text { with } \\
\text { Pauli-potential }\end{array}$ \\
\hline$\alpha\left(\mathrm{fm}^{-2}\right)$ & 0.25 & 0.1152 \\
$t_{1}\left(\mathrm{MeV} \mathrm{fm}^{3}\right)$ & -7264.04 & -84.5 \\
$t_{\gamma}(\mathrm{MeV} \mathrm{fm})^{6}$ & 87.65 & 188.2 \\
$\gamma$ & 1.676 & 1.46 \\
$V_{0}^{\text {Yuk }}(\mathrm{MeV} \mathrm{fm})$ & -0.498 & -85.1 \\
$\gamma_{Y}(\mathrm{fm})$ & 1.4 & 1.0 \\
$V_{0}^{\text {Pauli }}(\mathrm{MeV})$ & - & 98.95 \\
$q_{0}(\mathrm{fm})$ & - & 2.16 \\
$p_{0}(\mathrm{MeV})$ & - & 120 \\
\hline
\end{tabular}

Unfortunately, the generalization of such two body forces to the relativistic region is not a simple task and is not incorporated in the present UrQMD model. In principle, the interaction must be mediated by fields, which are propagated according to wave equations or one must make use of the so called constraint Hamiltonian dynamics [17,22]. Many other models avoid the propagation of fields using local density approximation. Above $2 \mathrm{~A} \mathrm{GeV}$, we therefore resort to cluster decomposition in phase space, i.e. potential interactions are only enforced for particles with relative momenta smaller than $2 \mathrm{GeV} / \mathrm{c}$.

\section{Cross- Section}

The cross- section is interpreted geometrically as an area. Two particles collide if their distance $d_{\text {trans }}$ fulfils the relation:

$$
d_{\text {trans }} \leq d_{0}=\sqrt{\frac{\sigma_{\text {tot }}}{\pi}}, \sigma_{\text {tot }}=\sigma(\sqrt{s}, \text { type })
$$

The total cross section $\sigma_{\text {tot }}$ depends on the center of mass, c.m. energy $\sqrt{s}$ and on the species and quantum numbers of the incoming particles. $d_{\text {trans }}$ is the relative distance between the two colliding particles (in three dimensional configuration space). At the point of closest approach, this distance is purely transverse with regard to the relative velocity vector of the particles. During the calculation, each particle is checked at the beginning of each time step whether it will collide according to criterion (3.1) within that time step. After each binary collision or decay the outgoing particles are checked for further collisions within the respective time step. The particles move in Minkowsky-space along 4-dimensional curved trajectories. At the beginning of each time step criterion (3.1) is applied to scan for collisions. Now, however, $d_{\text {trans }}$ is defined as the covariant relative distance:

$$
d_{\text {trans }}=\sqrt{\left(\frac{\left(q_{n}-q_{m}\right)^{v}\left(p_{m}-p_{n}\right)_{v}}{\left(p_{n}+p_{m}\right)^{2}}\left(p_{n}+p_{m}\right)^{\mu}-\left(q_{n}-q_{m}\right)^{\mu}\right)^{2}}
$$

using 4-vectors for the locations $q_{v}$ and momenta $p_{v}$ of the particles.

In the UrQMD model a $6 \mathrm{~N}+1$ dimensional phase-space has been used. We therefore use a different ansatz to minimize the frame-dependence of the collisions: The impact parameter of two colliding particles here also called $d_{\text {trans }}$ is well defined in the local rest-frame of the two particles. It corresponds to the relative distance between the two particles at the time of closest approach. In order to compute the time of closest approach of the two particles one always transforms into the local rest-frame of the two particles (using a $6 \mathrm{~N}+1$ dimensional phase space this implies that no time coordinates are transformed). With $\boldsymbol{q}_{i}$ being the locations and $\boldsymbol{p}_{i}^{\prime}$ the momenta in the local rest frame one obtains for the squared impact parameter $d_{\text {trans }}^{2}$ :

$$
\begin{gathered}
d_{\text {trans }}^{2}=d^{2}-d_{\|}^{2} \\
d_{\text {trans }}^{2}=\left(\boldsymbol{q}_{1}-\boldsymbol{q}_{2}\right)^{2} \frac{\left(\left(\boldsymbol{q}_{1}-\boldsymbol{q}_{2}\right) \cdot\left(\boldsymbol{p}_{1}^{\prime}-\boldsymbol{p}_{2}^{\prime}\right)\right)^{2}}{\left(\boldsymbol{p}_{1}^{\prime}-\boldsymbol{p}_{2}^{\prime}\right)^{2}}
\end{gathered}
$$

The constraint of always using the local rest frame of the colliding particles ensures that the cross section of the two particles always calculated in the same fashion and does not depend on the reference frame of the nucleus-nucleus collision. The time of closest approach $\tau_{\text {coll }}$ (i.e. the collision time), however, still depends on the reference frame of the nucleus-nucleus reaction. This dependence cannot be avoided since the system clock is linked to that reference frame. Therefore the time order of the individual binary collisions strongly varies with the respective reference frame [23]. Using the locations $r_{i}$ and the momenta $p_{i}$ in the reference frame of the nucleus-nucleus collision one obtains for the time of closest approach for the two colliding particles:

$$
\tau_{\text {coll }}=-\frac{\left(\boldsymbol{r}_{1}-\boldsymbol{r}_{2}\right) \cdot\left(\frac{p_{1}}{E_{1}}-\frac{p_{2}}{E_{2}}\right)}{\left(\frac{p_{1}}{E_{1}}-\frac{p_{2}}{E_{2}}\right)^{2}}
$$

The frame independent definition of the cross section (via the impact parameter in the two-particle rest frame) is an important factor in ensuring the approximate reference-frame independence.

\section{Results on Hadron-Hadron Cross-Sections}

The total cross-sections depend on nature of the interacting particles and on the geometrical interpretations in terms of the impact parameter. A collision between two hadrons will occur if $d<\sqrt{\sigma_{\text {tot }} / \pi}$, where $d$ and $\sigma_{\text {tot }}$ are the impact parameter of the hadrons and the total cross-section of the two hadrons, respectively. In the 
UrQMD model, the total cross-section $\sigma_{\text {tot }}$ depends on the isospins of colliding particles, their flavor, and the c.m. energy. In this section, we compare the UrQMD cross-sections with experimental data. The total baryon-baryon cross-section of the reaction $\mathrm{A}+\mathrm{C} \rightarrow \mathrm{D}+\mathrm{E}$ has the general form

$$
\sigma_{\text {tot }}^{B B}(\sqrt{s}) \propto\left(2 S_{D}+1\right)\left(2 S_{E}+1\right) \frac{\left\langle p_{D, E}\right\rangle}{\left\langle p_{A, C}\right\rangle} \frac{1}{s}|\mathcal{M}|^{2}
$$

with the spins of the particles, $\mathrm{S}_{\mathrm{i}}$, momenta of the pairs of particles, $\left\langle p_{i, j}>\right.$, in the two particle rest frame, and the matrix element $|\mathcal{M}|^{2}$. The matrix element $|\mathcal{M}|^{2}$, however, it is a function of all the particle's quantum numbers as well as its momenta and the c.m. energy.
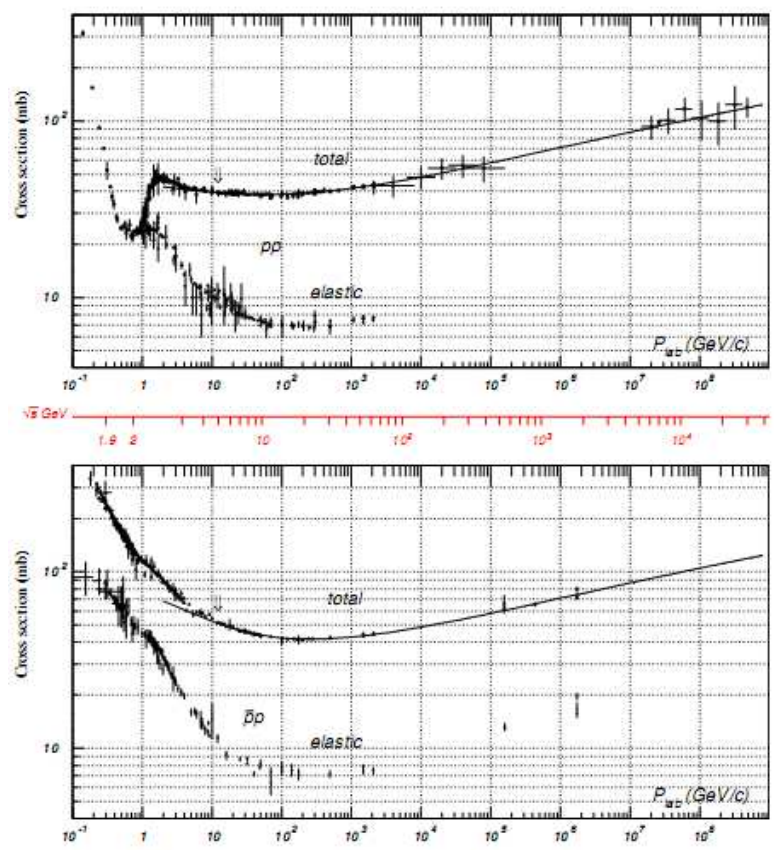

Fig 1. The total and elastic cross-section for $p p$ and $\bar{p} p$ collisions as a function of laboratory beam momentum and total center of mass energy.

The dependence total and inelastic cross-section for proton (anti) proton on the projectile momentum or the c.m. energy is shown in Fig. 1. The experimental data is obtained from Particle Data Group [24]. It is noticed that the total cross-section has no marked difference for values of momentum above $10^{4} \mathrm{GeV} / \mathrm{c}$ for $\mathrm{pp}$ and proton-antiproton while the values below $10^{4} \mathrm{GeV} / \mathrm{c}$ is projectile dependent.

The total cross-section for proton-proton collisions from a beam momentum $0.1 \mathrm{GeV}$ up to $10^{4} \mathrm{GeV}$ is shown in Fig. 2 . The total and inelastic cross-sections of the $p p$ reaction are well measured in this energy region [25]. The experimental values are comparing with the prediction of RQMD represent by solid line.

One finds a complex structure in this cross-section. Local minima are notice at $700 \mathrm{MeV} / \mathrm{c}\left(\mathrm{E}_{\mathrm{c} . \mathrm{m}} \approx 2 \mathrm{GeV}\right)$ and 100 $\mathrm{GeV} / \mathrm{c}\left(\mathrm{E}_{\mathrm{c} . \mathrm{m}} \approx 10 \mathrm{GeV}\right)$. The maximum at $2 \mathrm{GeV} / \mathrm{c}\left(\mathrm{E}_{\mathrm{c} . \mathrm{m}} \approx 2-\right.$ $3 \mathrm{GeV}$ ) and a rise is above $100 \mathrm{GeV} / \mathrm{c}$. Note that the steep rise in the data below $300 \mathrm{MeV} / \mathrm{c}$ is due to soft Coulomb interaction of the protons and is taken care of via the potential interaction. The total and elastic proton-proton and proton-neutron cross sections are well describing [25]. Since their functional dependence on $\sqrt{s}$ shows at low energies a complicated shape, UrQMD uses a table-lookup for those cross-sections. In addition, Fig. 2 shows the comparison between measurements for the proton-proton elastic cross sections and the respective UrQMD table lookup. The same comparison for the proton neutron case is shown in Fig. 3.
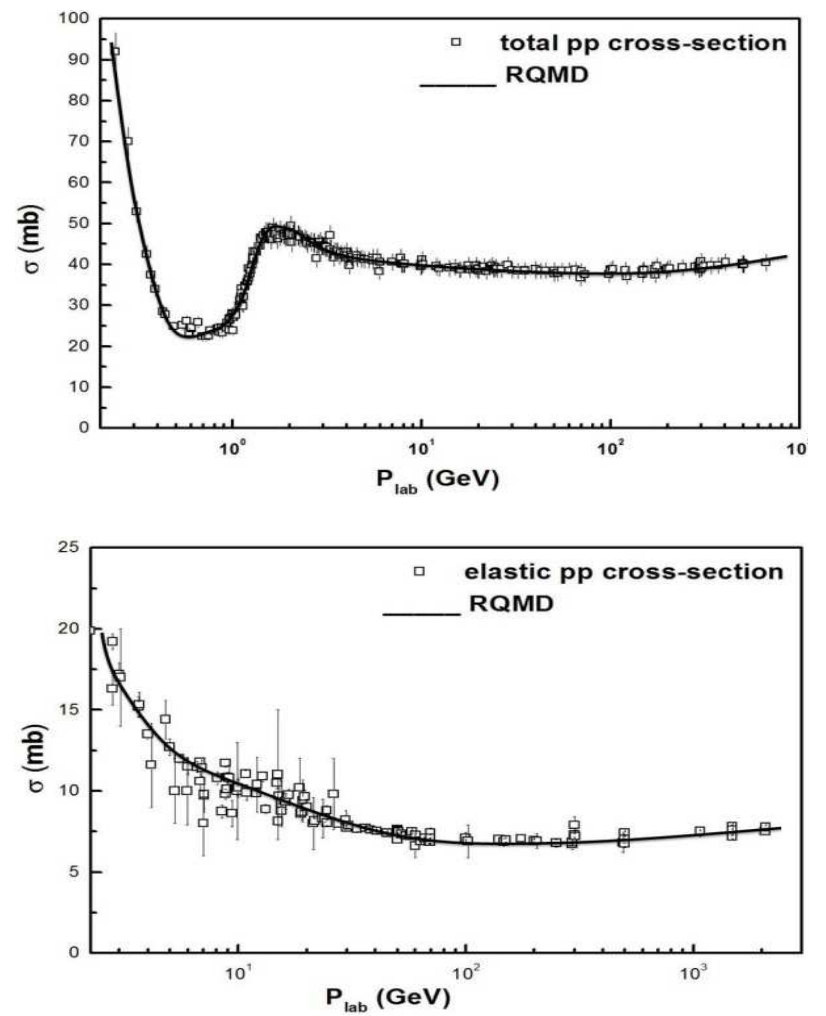

Fig 2. The above is total pp cross-section and below is the elastic $p p$ with the momentum of incident particle. The prediction of $R Q M D$ represent by the solid line.

At low energies, large differences are visible between the proton-proton and the proton-neutron cross sections. Therefore, the proper treatment of isospin especially at low energies is of major importance; simple averaged nucleon-nucleon cross-sections should not use. The neutron-neutron cross-section treated as equal to the proton-proton cross section (isospin-symmetry). In the high-energy limit $(\sqrt{s} \geq 5 \mathrm{GeV})$ the CERN/HERA parameterization for the proton-proton cross section is used [25].

Figure 4 shows the total and elastic cross-section $\pi^{-} p$ interactions as a function of momentum of the incident particle the solid line is the prdection of the RQMD. The model can gives a qualitative success for calculations of momentum dependece of cross-section. A similar conclusion can obtained for the same comparison but for $\pi^{+} p$ interactions. It reflect the fundamental assumptios for this model can give an enclusive description of this interactions and in this range of interactions momenta. The same behavior is for $k^{+} p$ interactions. It may be becaue the simmlarity for all mesons family. 

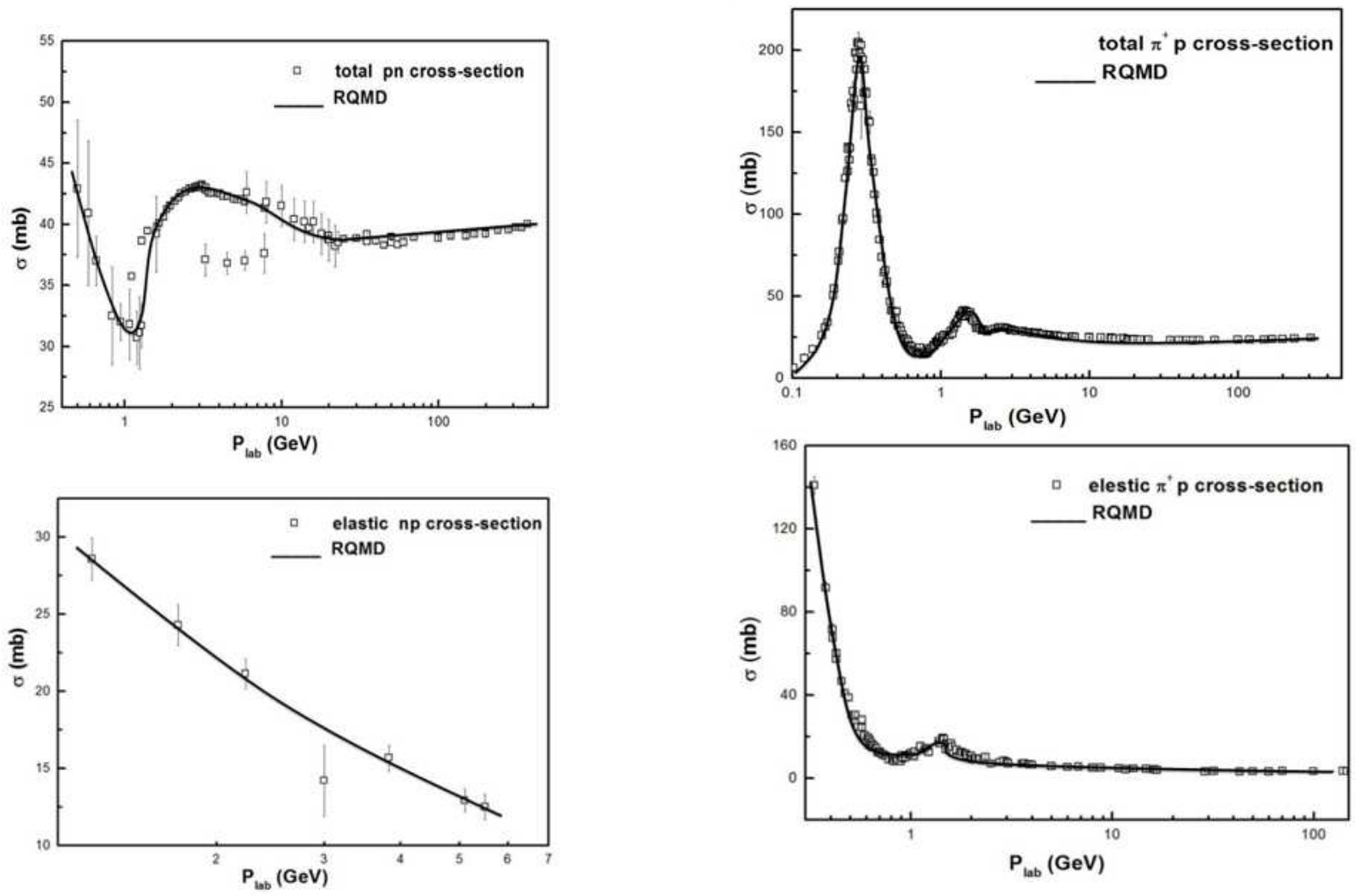

Fig 3. Above is total pn cross-section and below is the elastic $n p$ with the momentum of incident particle. The prediction of RQMD represent by the solid line.
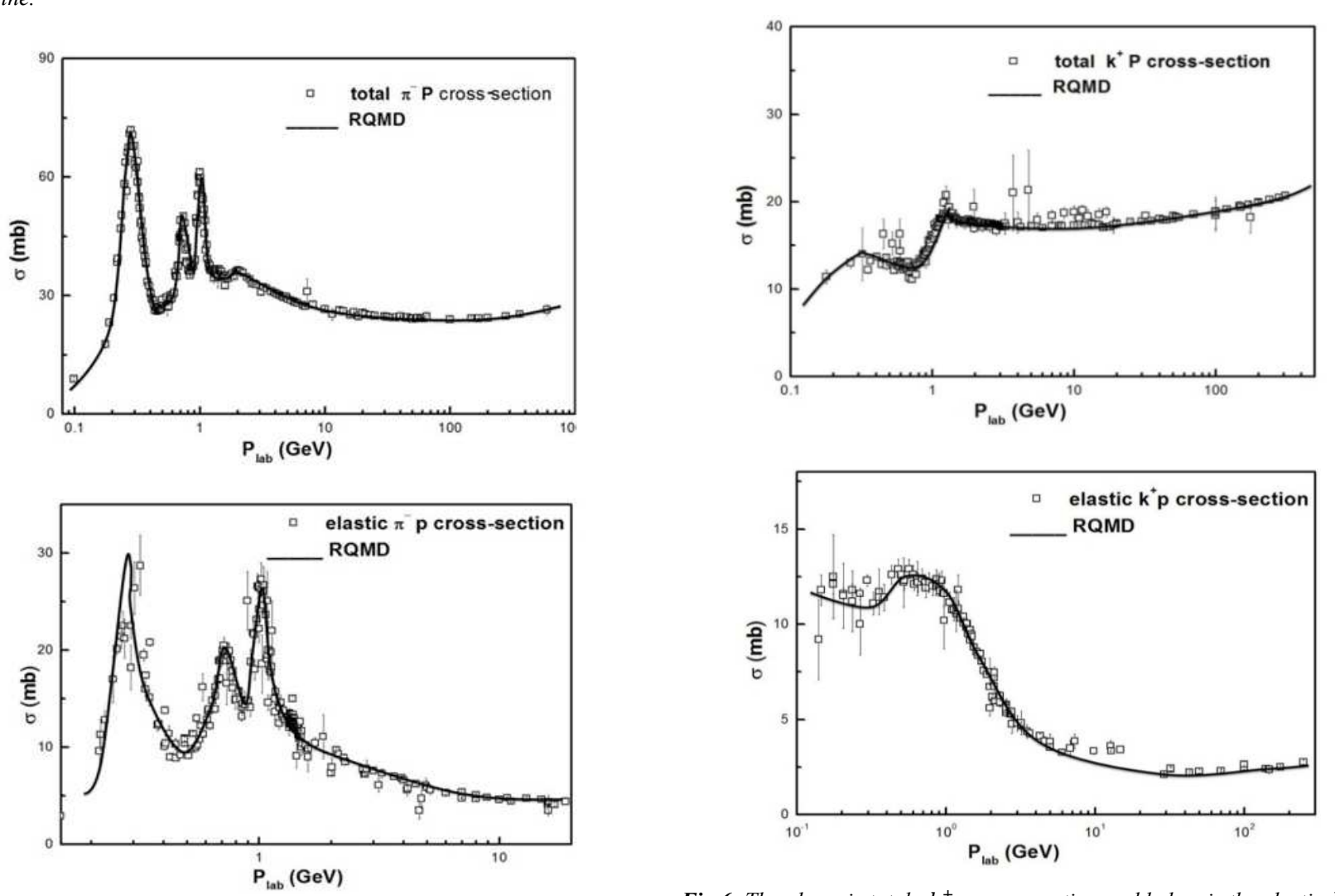

Fig 4. Above is total $\pi^{-} p$ cross-section and below is the elastic $\pi^{-} p$ with the momentum of incident particle. The prediction of RQMD represent by the solid line.

Fig 5. The above is total $\pi^{+} p$ cross-section and below is the elastic $\pi^{+} p$ with the momentum of incident particle. The prediction of RQMD represent by the solid line.

Fig 6. The above is total $k^{+} p$ cross-section and below is the elastic $k^{+} p$ with the momentum of incident particle. The prediction of RQMD represent by the solid line. 
At low energies $<2 \mathrm{GeV} / \mathrm{c}$ the structure in the $p p$ cross-section is mainly due to the inelastic channels and at higher energies, the contributions may be produce by a way, which give the excitation of color strings, which is the dominant process at high energies in this model. The total cross section in this region is give by the CERN-HERA parameterization [24] as shown in table 2. Partonic pQCD scattering is not included into the UrQMD model in the present version. The difference between the total and the elastic cross-section is taking as the inelastic cross-section.

Table 2. Parameters of the CERN-HERA fit [24] used in UrQMD for the total and elastic-cross-section above the resonance region $\left(p_{\text {lab }}>2 \mathrm{GeV} / \mathrm{c}\right)$. The cross sections are parameterized as: $\sigma_{\text {tot el }}(p)=A+B p^{n}+C \ln ^{2}(p)+D$ $\ln (p)$, with the laboratory momentum $p$ in $\mathrm{mb}$.

\begin{tabular}{cccccc}
\hline $\boldsymbol{\sigma}(\boldsymbol{m b})$ & $\mathbf{A}$ & $\mathbf{B}$ & $\mathbf{C}$ & $\mathbf{D}$ & $\mathbf{n}$ \\
\hline$p p($ total $)$ & 48.0 & 0 & 0.522 & -4.51 & 0 \\
$p p($ elastic $)$ & 11.9 & 26.9 & 0.169 & -1.85 & -1.21 \\
$p n($ total $)$ & 47.3 & 0 & 0.513 & -4.27 & 0 \\
$\bar{p} p($ total $)$ & 38.4 & 77.6 & 0.26 & -1.2 & -0.64 \\
$\bar{p} p($ elastic $)$ & 10.2 & 52.7 & 0.125 & -1.28 & -1.16 \\
$\pi^{+} p$ (total) & 16.4 & 19.3 & 0.19 & 0 & -0.42 \\
$\pi^{+} p($ elastic $)$ & 0 & 11.4 & 0.079 & 0 & -0.4 \\
$\pi^{-} p$ (total) & 33.0 & 14.0 & 0.456 & -4.03 & -1.36 \\
$\pi^{-} p($ elastic $)$ & 1.67 & 11.2 & 0.043 & 0 & -0.64 \\
$k^{+} p$ (total) & 18.1 & 0 & 0.26 & -1 & 0 \\
$k^{+} p($ elastic $)$ & 5.0 & 8.1 & 0.16 & -1.3 & -1.8 \\
\hline
\end{tabular}

\section{Conclusions}

1. For projectile momentum above $10^{4} \mathrm{GeV} / \mathrm{c}$ the total cross-section for $p p$ and $\bar{p} p$ collision is independent on the projectile momentum and energy in the center of mass system.

2. In energy range 0.1 up to $104 \mathrm{GeV}$ the total and elastic cross-section for $p p, p n, \pi^{-} p, \pi^{+} p, k^{-} p$ and $k^{+} p$ collisions are in good agreement with the predictions of the relativistic RQMD model.

3. There is a great importance for isotopic spin in low energy cross-section calculations.

4. RQMD model cross-section calculations give a good agreement results with experiments for $\pi^{-} p, \pi^{+} p$, $k^{-} p$ and $k^{+} p$ collisions. It may be due to the similarity of the internal structure of the mesons group.

\section{Acknowledgement}

The author wishes to thanks Particle Data Group PDG for the recent experimental data. Much appreciation to QMD group for allowed theoretical details and supplements the calculations program.

\section{References}

[1] Aamodt K. et al., Eur. Phys. J. C, 68 (2010) 345.

[2] Aad G. et al., Phys. Lett. B, 688 (2010) 21.

[3] Aad G. et al., New J. Phys., 13 (2011) 053033.

[4] Khachatryan V. et al., JHEP, 02 (2010) 041.

[5] Khachatryan V. et al., Phys. Rev. Lett., 105 (2010)022002.

[6] J. Aichelin, Phys. Rep. 202, 233 (1991).

[7] J. Aichelin, A. Rosenhauer, G. Peilert, H. St"ocker, andW. Greiner, Phys. Rev. Lett. 58, 1926 (1987).

[8] J. Aichelin and H. Stöcker, Phys. Lett. B176, 14 (1986).

[9] G. Peilert, A. Rosenhauer, J. Aichelin, H. Stöcker, and W. Greiner, Phys. Rev. C39, 1402 (1989).

[10] M. Bleicher et al., J. Phys. G 25 (1999) 1859 [arXiv:hep-ph/9909407].

[11] S. A. Bass et al., Prog. Part. Nucl. Phys. 41 (1998) 255 [Prog. Part. Nucl. Phys. 41 (1998)225] [arXiv:nucl-th/9803035].

[12] J. J. Molitoris, J. B. Hoffer, H. Kruse, and H. Stöcker, Phys. Rev. Lett. 53, 899 (1984).

[13] R. Bodmer and C. N. Panos, Phys. Rev. C15, 1342 (1977).

[14] L. Wilets, E. M. Henley, M. Kraft, and A. D. McKellar, Nucl. Phys. A282, 341 (1977).

[15] R. Bodmer, C. N. Panos, and A. MacKellar, Phys. Rev. C22, 1025 (1980).

[16] S. Kiselev and Y. Pokrovskil, Sov. J. Nucl. Phys. 38, 46 (1983).

[17] H. Sorge, H. Stöcker, and W. Greiner, Ann. Phys. 192, 266 (1989).

[18] C. Hartnack et al., Nucl. Phys. A538, 53c (1992).

[19] S. A. Bass, C. Hartnack, H. Stöcker, andW. Greiner, Phys. Rev. C51, 3343 (1995), nucl- th/9501002.

[20] C. Hartnack et al., Nucl. Phys. A495, 303 (1989).

[21] J. Konopka, Thesis, Goethe Universit"at, Frankfurt am Main, Germany (1996).

[22] D. G. Currie, T. F. Jordan, and E. C. G. Sudarshan, Rev. Mod. Phys. 35, 350 (1963)

[23] T. Kodama, S. B. Duarte, K. C. Chung, R. Donangelo, and R. A. M. S. Nazareth, Rio De Janeiro Pesquisas Fis. NF-83-026 (1983).

[24] Particle-Data-Group, Phys. Rev. D54 (1996).

[25] J. Beringer et al. (Particle Data Group), Phys. Rev. D86, $010001(201$ 